\title{
Capsule endoscopy diagnosis of radiation enteritis in a patient previously considered to have celiac sprue
}

A 65-year-old woman, who had been diagnosed with celiac sprue, presented with chronic diarrhea, fatigue, anorexia, and impaired night vision despite following a strict gluten-free diet. Diarrhea began 10 years earlier, around the time she underwent hysterectomy followed by pelvic radiation for endometrial cancer. Five years later, celiac sprue was diagnosed based on a positive gliadin IgG antibody and small-bowel biopsies revealing villous atrophy, crypt hyperplasia, and increased intraepithelial lymphocytes.

The patient's persistent symptoms, despite a 5-year gluten-free diet, prompted further evaluation. On physical exam she was a comfortable, well-developed, thin white female (body mass index [BMI] 17.2). She exhibited bitemporal wasting, and rectal exam was trace heme positive. Repeat gliadin antibodies revealed a positive $\operatorname{IgG}(106 ;<20$ negative) and negative IgA. Tissue transglutaminase IgG and IgA, and endomysial IgA, were negative. Serum IgA was normal. Human leukocyte antigen (HLA)-DQ2 and HLA-DQ8 were negative. Upper endoscopy revealed mild scalloping and duodenal nodularity. Small-bowel biopsies were consistent with celiac sprue with villous blunting and numerous intraepithelial lymphocytes (Figure $\mathbf{1}$ ). Wireless capsule endoscopy confirmed scalloping and nodularity in the proximal small bowel (Video $\mathbf{1}$ ). The distal small bowel (Video 2 ) was notable for marked edema, erythema, and neovascularization. A colonoscopy to the cecum demonstrated minimal right colon and rectal telangiectasias. A diagnosis of radiation enteritis was established.

Celiac sprue was originally diagnosed based on small-bowel histology that was characteristic, but not specific, for this disease [1]. The patient also had a positive gliadin IgG antibody, which is the least specific of the celiac antibodies (sensitivity $80 \%$, specificity $80 \%$ ) [2]. Upon reevaluation, the combined result of negative serological findings and absence of HLA-DQ2 or HLA-DQ8 was approximately
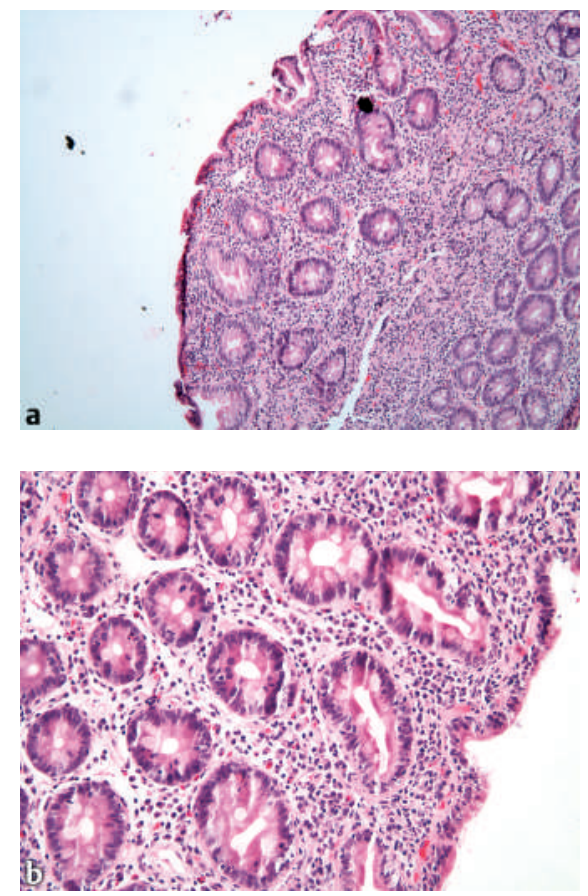

Figure 1 Histological appearance of duodenal biopsy specimen. a Marked blunting of the villi is seen (hematoxylin and eosin [H\&E] stain; $\times 10$ ) and, b an increased number of intraepithelial lymphocytes in the crypts and surface epithelium (H\&E stain; $\times 20$ ).

$98 \%$ specific for excluding celiac sprue $[3$, 4].

The new technology of wireless capsule endoscopy was crucial in establishing an accurate diagnosis of radiation enteritis, a disorder that can be similar to celiac sprue in both clinical and histological presentation.

Endoscopy_UCTN_Code_CCL_1AC_2AZ

Endoscopy_UCTN_Code_CCL_1AC_2AD

Endoscopy_UCTN_Code_TTT_1AP_2AB

\section{A. Jazwinski, J. Palazzo, D. Kastenberg} Jefferson Medical College, Philadelphia, Pennsylvania, USA.

\section{References}

${ }^{1}$ Farrell R, Kelly C. Diagnosis of celiac sprue. Am J Gastroenterol 2001; 96: 3237-3246

2 Rostom A, Dube C, Cranney A et al. Celiac disease. Evidence Report/Technology Assessment No. 104. AHRQ publication no. 04E029-2. Rockville, MD: Agency for Healthcare Research and Quality 2004

${ }^{3}$ Green PH, Jabri B. Coeliac disease. Lancet 2003; 362: 383-391

${ }^{4}$ Sollid LM, Markussen G, Ek J et al. Evidence for a primary association of celiac disease to a particular HLA-DQ $\alpha / \beta$ heterodimer. J Exp Med 1989; 169: 345-350

Corresponding author

\section{Kastenberg, M.D.}

132 South 10th Street

Philadelphia

Pennsylvania 19107

USA

Fax: $\quad+1-215-503-2578$

Email: david.kastenberg@jefferson.edu

\section{Video 1}

Proximal jejunum: scalloping and nodularity.

\section{Video 2}

lleum: features consistent with radiation enteritis - edema, erythema, and neo-vascularisation.

online content including video sequences viewable at:

www.thieme-connect.de/ejournals/abstract/ endoscopy/doi/10.1055/s-2006-944715 